Check for updates

Cite this: RSC Adv., 2019, 9, 39958

\title{
Highly sensitive strain sensors based on hollow packaged silver nanoparticle-decorated three- dimensional graphene foams for wearable electronics $\dagger$
}

\author{
Xinxiu Wu,,$^{a}$ Fangfang Niu,,$^{\mathrm{b}}$ Ao Zhong, ${ }^{\mathrm{a}}$ Fei Han, ${ }^{\mathrm{c}}$ Yun Chen, ${ }^{\mathrm{d}}$ Jinhui Li, (DD *a \\ Guoping Zhang, (D) *a Rong Sun $\mathbb{D}^{\mathrm{a}}$ and Ching-Ping Wong ${ }^{\mathrm{e}}$
}

Flexible strain sensors possess a great potential for applications in wearable electronic devices for human motion detection, health monitoring, implantable medical devices and so on. However, the development of highly sensitive strain sensors remains a challenge in the field of wearable electronics. Herein, we prepared a highly sensitive strain sensor, which was composed of a three-dimensional reduced graphene oxide foam decorated with silver nanoparticles (Ag NPs) to enhance the conductivity. Then, half-cured polydimethylsiloxane was employed to get a special "hollow packaged" structure. Thanks to the synergistic conductive effect of Ag NPs and the reduced graphene oxide flakes as well as the special "hollow packaged" structure, the as-prepared flexible strain sensor not only possessed a dramatic gauge factor of 1588 (at $50 \%$ sensing strain), but also exhibited high stability in 500 cycles of $30 \%$ strain. The mechanism of the enhancement of the sensitivity with the special "hollow packaged" structure was discussed as well. Meanwhile, the detection of the bending and rotation of wrists and the bending of fingers and arms was demonstrated, showing attractiveness in human motion detection.

Received 21st October 2019

Accepted 18th November 2019

DOI: $10.1039 / c 9 r a 08118 f$

rsc.li/rsc-advances flexibility, stretchability, biocompatibility, high sensitivity, high durability, rapid response, and so on. ${ }^{6,7}$ In addition, the resistance of most sensors varies due to the change in conductive paths. Basically, there are two typical ways to increase the sensitivity of these sensors. ${ }^{8}$ First, the conductive materials have been designed as porous structures, for example, 3D graphene foams or porous elastomer polymers coated with conductive nano-materials. ${ }^{9}$ Due to these porous structures, the sensors can generate more variations in conductive paths, thereby increasing the resistance change and sensitivity more significantly. Second, microstructures are fabricated on the contact surfaces of the conductive paths. ${ }^{10}$ For example, cracks change due to the simultaneous changes in body resistance and contact resistance, and the sensitivity of the sensor can be significantly improved. Prospective strain sensors combine multidimensional conductive materials to form a conductive network, which include metal particles, carbon black, ${ }^{11}$ carbon nanotubes, ${ }^{12}$ silver nanowires, and graphene. ${ }^{13}$

Graphene is an atomic-scale, thin, two-dimensional hexagonal honeycomb material. ${ }^{14}$ Its mechanical stiffness, strength and elasticity, electrical conductivity and thermal conductivity are noteworthy. ${ }^{15,16}$ In addition, due to the flexibility and biocompatibility, graphene can be a promising material for high-performance, multifunctional wearable electronics and biomedical applications. ${ }^{17,18}$ However, the application of graphene in practical wearable electronics is challenging due to its 
electrical properties under high tensile conditions., ${ }^{7,15,19}$ Chen et al. manufactured 3D interconnected graphene networks by chemical vapor deposition, embedding these networks in a polydimethylsiloxane (PDMS) matrix to produce strain sensors capable of withstanding tensile strains up to $95 \%$ and providing a gauge factor of $2 .^{20}$ Based on Chen's work, a layer of polyethylene terephthalate (PET) was introduced to form a double-layered graphene foam/PDMS-PET composite material, and its bending sensitivity was increased by a factor of 6 . After the 3D graphene foam was made into small fragments of 200-300 $\mu \mathrm{m}$, a sensitivity factor of 15-29 or even higher was obtained at $70 \%$ strain. ${ }^{21}$ Our group reported a 3D graphene foam formed by the reduction and self-assembly of graphene oxide, which was then infiltrated with PDMS to obtain a sensitivity factor of 98.66 at $5 \%$ strain and a sensitivity factor of 47.74 at $20 \%$ strain. $^{22}$ However, none of these graphene-polymer composites exhibited a large working strain range and high sensitivity at the same time, which is essential for fine and coarse motion detection.

The combination of metal nanoparticles and graphene has been employed in the applications of flexible sensors, chemical sensors, fuel cells, catalysts, etc. ${ }^{23,24}$ As a kind of zerodimensional nano-material, Ag nanoparticles (Ag NPs) have many advantages, like a simple preparation process and excellent electrical properties, which can improve the performance of electronic devices through decoration. ${ }^{25}$ Therefore, more and more attention has been paid to Ag NPs. Zhang et al. ${ }^{26}$ reported an efficient, simple and repeatable piezoresistive sensor based on a carbon nanotube@silver nanoparticle (CNT@AgNP) homogeneous 3D hybrid network. Highly elastic, sensitive and wearable sensors were obtained by using the porous structure of a sponge and the synergistic effect of CNTs/Ag NPs. Yang et al. developed a $\mathrm{Ag}$ NP (Ag nanoparticle)-bridged graphene strain sensor. Ag NPs served as a bridge connecting the selfoverlapping graphene sheets, which endowed the sensor with high sensitivity of 475 at the strain of $14.5 \% .^{27}$ Our group invented a high-sensitivity, stretchable and conductive strain sensor with a sensitivity of 107 at $120 \%$ strain. This high sensitivity was due to the dual synergistic effect of designing fragmentized graphene sponge structures and $\mathrm{Ag}$ NPs. ${ }^{28}$

Based on the above-mentioned considerations, we designed a novel strain sensor, which was composed of a threedimensional graphene foam decorated with Ag NPs and packaged with PDMS. The graphene foam decorated with Ag NPs (GF/Ag) was prepared by a two-step reduction method and then encapsulated with two semi-cured PDMS matrixes. The obtained strain sensors possessed a special "hollow packaged" structure with high sensitivity and stability for highperformance wearable strain sensors.

\section{Experimental section}

\section{Materials}

Graphite powder, polyvinylpyrrolidone (PVP), and ascorbic acid were supplied by Aladdin (Shanghai, China). The chemicals potassium permanganate $\left(\mathrm{KMnO}_{4}\right)$, sodium nitrate $\left(\mathrm{NaNO}_{3}\right)$, concentrated sulfuric acid $\left(\mathrm{H}_{2} \mathrm{SO}_{4}, 98 \%\right)$, and hydrochloric acid
( $\mathrm{HCl})$ were of reagent grade and purchased from Sinopharm Chemical Reagent Beijing Co., Ltd. PDMS prepolymer and the curing agent were supplied as two-part liquid component kits from Dow Corning (Sylgard 184). Silver nitrate $\left(\mathrm{AgNO}_{3}\right)$ and ammonia solution were purchased from LingFeng of Shanghai. Anhydrous ethanol was supplied by Sinopharm Group Chemical Reagent Co., Ltd.

\section{Preparation of the silver nanoparticle-decorated graphene foam}

Graphene oxide (GO) was prepared from natural graphite powders using the modified Hummers' method that can be found in our previous work. ${ }^{29}$ Graphene foams (GF) decorated with Ag NPs (GF/Ag) were synthesized via two steps. First, silver nitrate was reduced to Ag NPs on the surface of GO under the combined action of anhydrous ethanol and PVP. In a typical process, $20 \mathrm{~mL}$ of $\mathrm{GO}$ solution $\left(1 \mathrm{mg} \mathrm{mL}^{-1}\right)$ was sealed in a $30 \mathrm{~mL}$ glass bottle with the addition of $4 \mathrm{~mL}$ anhydrous ethanol solution with $2 \mathrm{mg} \mathrm{AgNO}_{3}$ at $60{ }^{\circ} \mathrm{C}$. Second, $0.16 \mathrm{~g}$ of ascorbic acid was added to a $30 \mathrm{~mL}$ glass bottle (the mass ratio of GO to ascorbic acid was $1: 8$ ). After standing at $90{ }^{\circ} \mathrm{C}$ for $3 \mathrm{~h}$, the bottle was cooled freely and a graphene hydrogel decorated with Ag NPs was obtained. The hydrogel was washed with deionized water for several times and treated in ammonia solution at $90{ }^{\circ} \mathrm{C}$ for $1 \mathrm{~h}$ in a sealed glass bottle. In addition, the $\mathrm{GF} / \mathrm{Ag}$ foams were obtained after the freeze-drying process. Other samples were prepared with different mass ratios of GO to $\mathrm{AgNO}_{3}(30: 1,20: 1,10: 1$ and $5: 1)$ according to the abovementioned procedure. In contrast, pure GF was prepared without the addition of $\mathrm{AgNO}_{3}$. The foams with $30: 1,20: 1$, $10: 1$, and $5: 1$ ratios were designated as GF/Ag-1, GF/Ag-2, GF/ $\mathrm{Ag}-3$, and $\mathrm{GF} / \mathrm{Ag}-4$, respectively.

\section{Preparation of the 3D GF/Ag-based flexible strain sensor}

$\mathrm{GF} / \mathrm{Ag}$ composites were cut into a $20 \mathrm{~mm}$ long, $2 \mathrm{~mm}$ wide and $1 \mathrm{~mm}$ thick strip as a conductive material of the strain sensor. Both ends of the strip of GF were connected with copper wires using silver paste and cured at $80{ }^{\circ} \mathrm{C}$ for $1 \mathrm{~h}$. Then, certain amounts of the PDMS prepolymer and curing agent (mass ratio of $10: 1$ ) were mixed and degassed in vacuo, followed by pouring PDMS into a Teflon mold ( $1 \mathrm{~mm}$ depth) and curing at $70{ }^{\circ} \mathrm{C}$ for 15 min to obtain half-cured PDMS. Then, the GF/Ag composites were packaged with two half-cured PDMS films. In addition, the samples were heated at $70{ }^{\circ} \mathrm{C}$ for $4 \mathrm{~h}$ in order to cure the two pieces of semi-cured PDMS, after which the strain sensor of GF/ Ag/PDMS could be obtained. The $30: 1,20: 1,10: 1$, and $5: 1$ ratios of the GF/Ag composites encapsulated with PDMS were referred to as GF/Ag/PDMS-1, GF/Ag/PDMS-2, GF/Ag/PDMS-3, and GF/Ag/PDMS-4, respectively.

\section{Measurements}

Fourier transform infrared (FTIR) spectra were recorded using a Bruker Vertex 70 spectrometer (Bruker Optik GmbH, Ettlingen, Germany) in the range of $4000-400 \mathrm{~cm}^{-1}$. The morphology and microstructure were investigated by scanning electronic micrographs (SEM, nanoSEM 450, NOVA, USA). Powder X-ray 
diffraction (XRD) patterns were recorded using an X-ray diffractometer (RiGSKu D/Max 2500) equipped with a monochromated $\mathrm{Cu} \mathrm{K} \alpha$ radiation source $(\lambda=1.54 \AA)$ at a scanning rate of $2^{\circ} \mathrm{min}^{-1}$. The Raman spectra were measured using a LabRAM HR Raman Spectrometer (HORISA Jobin-Yvon, France) with a laser at the excitation wavelength of $632.8 \mathrm{~nm}$ and $15.7 \mathrm{~mW}$ power irradiation. The tensile test was carried out using a stretching machine (AG-X Plus 100N) at room temperature at a speed of $1 \mathrm{~mm} \min ^{-1}$.

\section{Results and discussion}

\section{Fabrication of the strain sensor}

The simple fabrication process of the GF/Ag composite is schematically illustrated in Fig. 1a. Compared with pure GF, $\mathrm{Ag}$ nanoparticles were introduced to increase the conductivity of the 3D foam, which then enhanced the sensitivity of the GF/ Ag/PDMS-based strain sensors. Ascorbic acid was employed to reduce not only GO but also silver nitrate, which was partially reduced with anhydrous ethanol. Anhydrous ethanol was used to reduce silver ions to Ag NPs with the help of PVP at first to avoid agglomeration. PVP can not only control the particle size of Ag NPs, but also combine with silver ions to form complexes that reduce its chemical potential. ${ }^{30}$ In addition, it was found that the heating process could speed up the reduction reaction process and the reaction could be completed in a short time. The preparation of the GF/Ag composites was simple and feasible. The reduction agents are non-toxic and odorless and environmentally friendly. The preparation process of the GF/ Ag/PDMS strain sensors is shown in Fig. 1b. It should be noted that this method is different from the typical infiltration and curing process of an elastomer, and it results in a "hollow packaged" structure, which then increases the sensitivity dramatically.

The morphological changes of GO, GF and GF/Ag composites were observed using SEM images at first. It can be seen from Fig. $\mathrm{S} 1 \uparrow$ that the GO sheet is relatively smooth. An aqueous solution containing only GO was reduced by ascorbic acid, and there were no nanoparticles on the wrinkled GF sheets

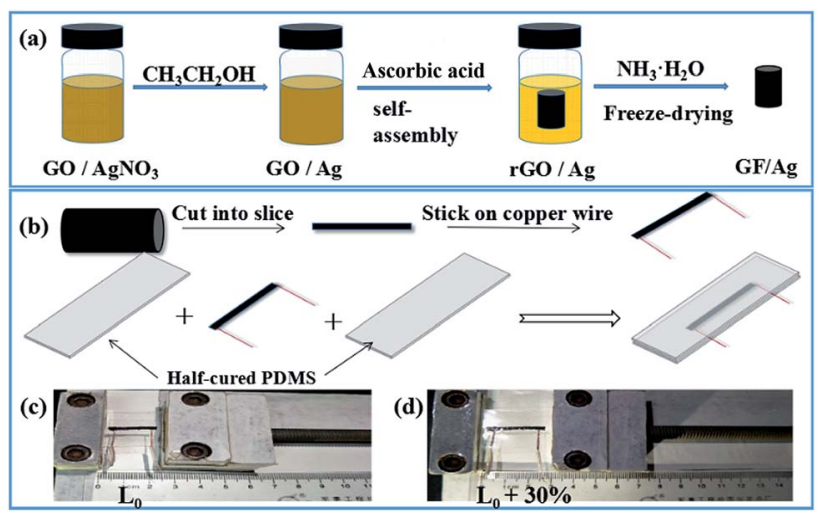

Fig. 1 (a) Preparation process of GF/Ag composites, (b) preparation of GF/Ag/PDMS strain sensors, (c) photographs of GF/Ag/PDMS sensors, (d) photographs of GF/Ag/PDMS sensors at a strain of $30 \%$.
(Fig. S1b $\dagger$ ). GF showed a 3D porous structure, as exhibited in Fig. S1c. $\uparrow$ Moreover, with the addition of $\mathrm{AgNO}_{3}$, lots of $\mathrm{Ag}$ NPs were attached to GO flakes after reduction with anhydrous ethanol, as shown in Fig. 2. Fig. S2a † shows the mapping of GO decorated with Ag NPs. From the energy spectrum, we can also see that Ag nanoparticles have actually grown on the GO flakes, as shown in Fig. S2b. $\dagger$

As shown in Fig. 3, the SEM images of the GF/Ag composites having four different ratios at different magnifications prove that the larger the proportion of silver nitrate added, the more the silver particles on GF. The EDS diagrams of the four proportions of the GF/Ag composites are shown in Fig. S3, $\dagger$ and all of them prove the existence of silver particles. Fig. $3 g$ and $h$ show the SEM images of the inner part of GF/Ag-4 at different magnifications. At a ratio of $5: 1$, more uniform Ag NPs can also be obtained in the inner part of $\mathrm{GF} / \mathrm{Ag}-4$. However, the mapping diagram of the bottom of GF/Ag-4 (Fig. S4a $\dagger$ ) shows that silver is indeed agglomerated, which means that Ag NPs are in excess for the graphene foam. Moreover, the energy spectrum (Fig. S4b $\dagger$ ) shows that the aggregated material on the graphene sheets is silver. The bottom of GF/Ag-4 is agglomerated with a lot of silver, which means that the amount of silver nitrate should not be too large.

Then, the chemical conditions of the as-prepared GF/Ag composites were checked by FTIR at first, as shown in Fig. 4a. The broad peak at $3390 \mathrm{~cm}^{-1}$ in the FTIR pattern is assigned to the stretching vibration of the $-\mathrm{OH}$ group, and the peak at $1738 \mathrm{~cm}^{-1}$ is ascribed to the $\mathrm{C}=\mathrm{O}$ stretching vibration. The $\mathrm{C}=\mathrm{C}$ stretching vibration peak appears at $1614 \mathrm{~cm}^{-1}$, and the peak at $1076 \mathrm{~cm}^{-1}$ is attributed to the $\mathrm{C}-\mathrm{O}$ stretching vibration. ${ }^{31,32}$ Compared with the observation for GO, it can be seen that the intensities of the peaks of $-\mathrm{OH}, \mathrm{C}=\mathrm{O}$ and $\mathrm{C}-\mathrm{O}$ of the GF/Ag composites sharply decrease or even disappear. This is because these groups are reduced by ascorbic acid. As shown in Fig. $4 \mathrm{~b}$, the peak of GO in the XRD pattern is $2 \theta=11^{\circ}$, and the peak of these GF/Ag composites is $2 \theta=24^{\circ}$. The GO spectrum shows a characteristic GO peak due to the oxygen-containing functional groups at about $11^{\circ}$, which corresponds to a relatively large interlayer distance. After GO is reduced, the amount of oxygen-containing functional groups decreases and the distance between the layers decreases. Therefore, GF/Ag shows a broad peak at about $24^{\circ}$. The diffraction peaks appearing at $2 \theta$ $=38.1^{\circ}, 44.2^{\circ}, 64.3^{\circ}$ and $77.4^{\circ}$ are attributed to the facecentered cubic (111), (200), (220) and (311) faces of Ag NPs.

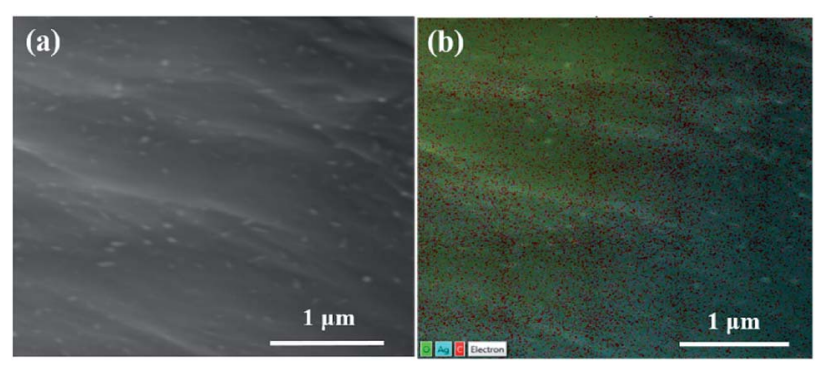

Fig. 2 (a) SEM image and (b) mapping diagram of GF/Ag-3. 


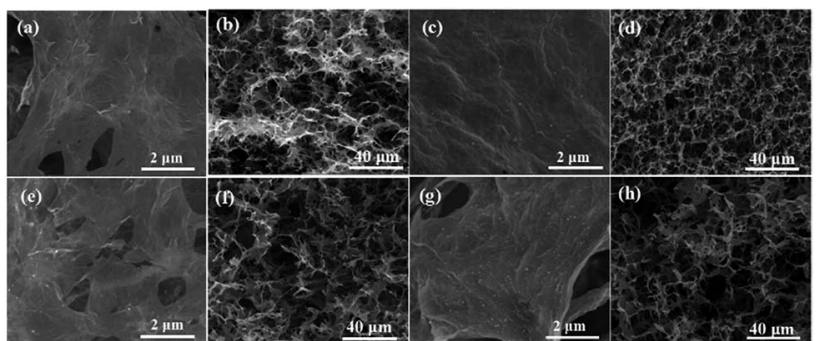

Fig. 3 SEM images of ( $a$ and b) GF/Ag-1, (c and d) GF/Ag-2, (e and f) GF/Ag-3, and (g and h) GF/Ag-4 at different magnifications.
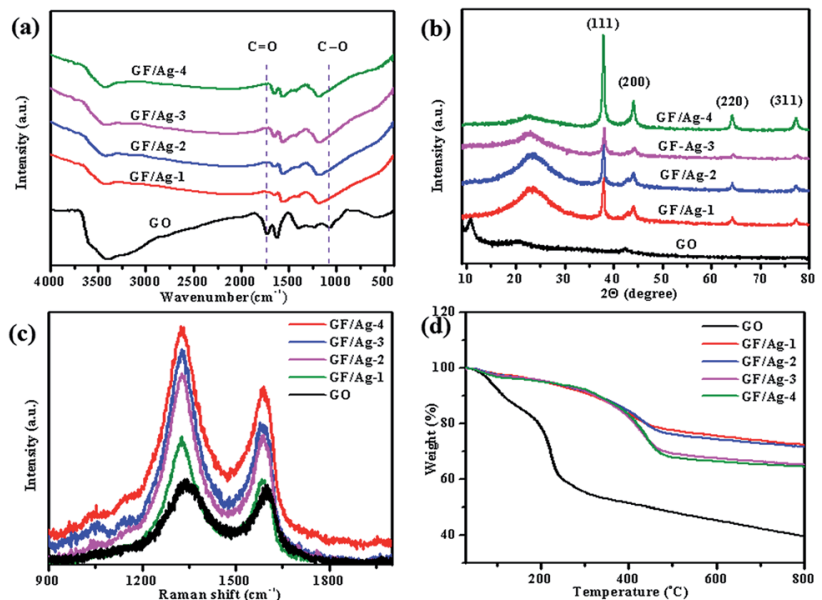

Fig. 4 (a) Infrared spectra, (b) XRD spectra, (c) Raman spectra graph, and (d) TGA graph of GO and GF/Ag NP composites.

Raman spectroscopy is a useful tool for the analysis of carbon materials. The Raman spectra of GO and the GF/Ag composites show two significant peaks near $1328 \mathrm{~cm}^{-1}$ and $1588 \mathrm{~cm}^{-1}$ (Fig. 4c). They are the D-band and G-band of carbon materials. The $I_{\mathrm{D}} / I_{\mathrm{G}}$ band intensity ratios of the $\mathrm{GF} / \mathrm{Ag}$ composites at $30: 1,20: 1,10: 1$, and $5: 1$ were 1.46, 1.49, 1.49 , and 1.34 , respectively. The $I_{\mathrm{D}} / I_{\mathrm{G}}$ band intensity ratio of GO was 1.08. An increase in the $I_{\mathrm{D}} / I_{\mathrm{G}}$ ratio indicates an increase in the number of $\mathrm{sp}^{2}$ domains in the GO aerogel, which confirms that GO is reduced. The $I_{\mathrm{D}} / I_{\mathrm{G}}$ values of GO were lower than the $I_{\mathrm{D}} / I_{\mathrm{G}}$ values of the $\mathrm{GF} / \mathrm{Ag}$ composites, indicating that GO was reduced with different Ag NP contents.

In addition to the above-mentioned analysis, the TGA curves for the ratios of the GF/Ag composites are shown in Fig. $4 \mathrm{~d}$, which prove a slower downward slope compared to that for GO; this means that the thermal stability is due to the removal of the oxygen-containing groups. GO weight loss occurs mainly at two temperatures: at about $100{ }^{\circ} \mathrm{C}$ and another at about $200{ }^{\circ} \mathrm{C}$. GO is hydrophilic and internally embeds water molecules. The evaporation of the adsorbed water leads to weight loss at around $100{ }^{\circ} \mathrm{C}$. Due to the decomposition of the oxygen-containing functional groups such as $\mathrm{C}-\mathrm{O}$ and $\mathrm{C}=\mathrm{O}$, the weight loss occurs at about $200{ }^{\circ} \mathrm{C}$. The GF/Ag composite is more hydrophobic than GO; thus, it has a lower weight loss at about $100{ }^{\circ} \mathrm{C}$.
The weight loss in the GF/Ag composite is only half that of GO, indicating that some of the oxygen-containing functional groups are removed during the reduction process. The total weight loss of the GF/Ag composite is about $25 \%$, while the total weight loss of GO is about $40 \%$. In summary, all of the abovementioned qualitative analyses demonstrated that GO was reduced by ascorbic acid.

In order to get the flexible and stretchable strain sensor, the GF/Ag composites were packaged with two half-cured PDMS films. The SEM images of the as-prepared GF/Ag/PDMS strain sensors are shown in Fig. 5. It can be seen that the as-prepared strain sensor possessed a special "hollow packaged" structure. In addition, the GF/Ag composites with the porous structure were found in the central part (Fig. 5a and b) and PDMS covered the surrounding area (Fig. $5 \mathrm{c}$ and d). This structure was completely different from that obtained after the infiltration of elastomers and possessed special properties for stretch strain sensing.

The mechanical properties of the GF/Ag samples were tested by compression cycles at first. Fig. 6a proves the stressstrain curves with stress loaded along the honeycomb pore axis direction at $50 \%$ strain. The stress values of GF/Ag-1 to GF/Ag-4 were $7.82 \mathrm{kPa}, 10.23 \mathrm{kPa}, 11.87 \mathrm{kPa}$ and $6.73 \mathrm{kPa}$ under the strain of $50 \%$, respectively. The results proved that the introduction of Ag NPs could increase the mechanical strength at first and then decrease because of the excess loading of Ag NPs, which destroyed the stability of the 3D structure of GF. Furthermore, Fig. 6b shows the relative resistance changes of the GF/Ag/PDMS strain sensors at 50\% strain. Compared to the observations for the GF/PDMS sensors (Fig. S5 $\dagger$ ), it can be seen that the relative resistance changes of the GF/Ag/PDMS sensors are much larger at 50\% strain, which indicates that the sensors have higher sensitivity. In addition, the resistance change increased with the addition of Ag NPs from GF/Ag/PDMS-1 to GF/Ag/PDMS-3 and then decreased. Besides, the details showed that the change in relative resistance before $25 \%$ strain was relatively low and then dramatically increased after $25 \%$. The calculation formula for the gauge factor is shown here: gauge factor $=$
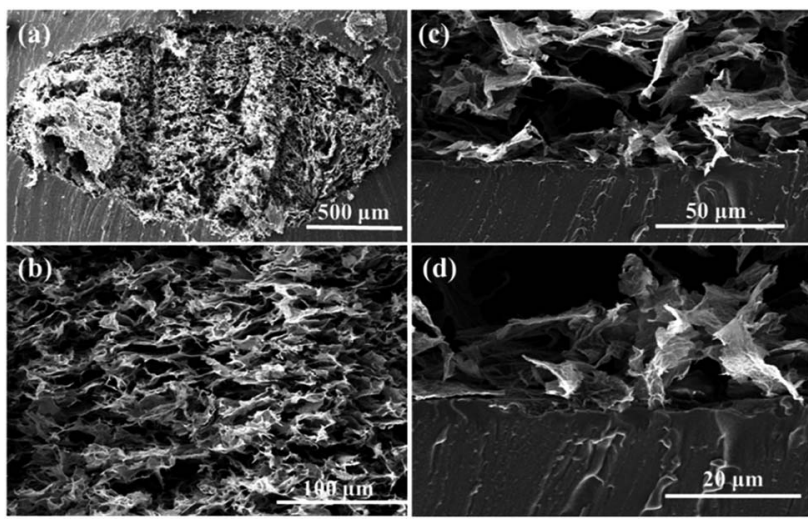

Fig. 5 Cross-sectional views of GF/Ag/PDMS at different magnifications ( $a$ and b). (c) Cross-sectional view at the boundary of GF/Ag/ PDMS and the enlarged view (d). 

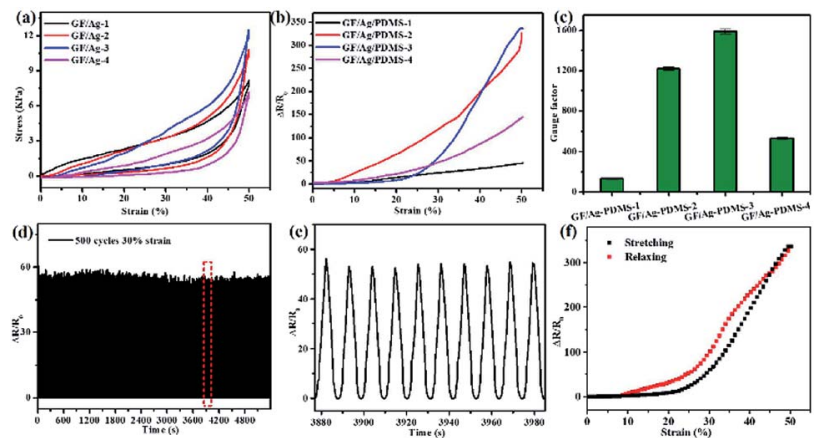

Fig. 6 (a) Stress-strain curves for a 50\% strain compression recovery of GF/Ag composites. (b) Curves of the relative resistance change and strain for GF/Ag/PDMS sensors. (c) Sensitivity factors for different proportions of the sensor at $50 \%$ strain. (d) 500 stretch-release cycles for GF/Ag/PDMS-3 at 30\% strain. (e) Relative resistance change curves of the strain sensor extracted from the red part in (d). ( $f$ ) Relative change in resistance versus strain for GF/Ag/PDMS-3.

$\left(\Delta R / R_{0}\right) / \varepsilon$; here, $\Delta R, R_{0}$ and $\varepsilon$ represent the changes in resistance, resistance without strain, and strain, respectively. From Fig. 6c, we can see that the gauge factors of GF/Ag/PDMS-1, GF/ Ag/PDMS-2, GF/Ag/PDMS-3, and GF/Ag/PDMS-4 are 131, 1220, 1588, and 534, respectively. Moreover, from GF/Ag/PDMS-1 to GF/Ag/PDMS-3, the content of Ag NPs increased gradually, and the gauge factor of the GF/Ag/PDMS strain sensors also increased accordingly. However, with the further increase in the proportion of $\mathrm{Ag}$ NPs $(5: 1)$, the gauge factor of $\mathrm{GF} / \mathrm{Ag} /$ PDMS-4 decreased. The possible reason is that with the further increase in the proportion of Ag NPs, the over-loaded Ag NPs could not be attached to the graphene foam network completely. Ag NPs were not uniformly dispersed, which was found to affect the conductivity of the graphene foam. Therefore, the gauge factor of the GF/Ag/PDMS strain sensors decreased from 1588 to 534 .

Compared with all the GF/Ag/PDMS-based sensors, as shown in Fig. 6a, the blue line (GF/Ag/PDMS-3) proves the best mechanical property with the largest compression strength with relatively abundant Ag NPs and reasonable micro-structures (compared with GF/Ag/PDMS-4, which possessed much more $\mathrm{Ag}$ NPs but lower strength at the compression of 50\%). The blue curve is negative (a smaller gauge factor) before $25 \%$ because of the abundant Ag NPs, which would result in more conductive paths during the compression with almost the same number of cracks generated during the stretch (compared with GF/Ag/ PDMS-1 and GF/Ag/PDMS-2). At the same time, GF/Ag/PDMS-4 possessed too much Ag NPs (as shown in Fig. S4 $\dagger$ ); moreover, there were fewer resistance changes during the compression process at a stretch strain below $25 \%$ and almost the same cracks changed during the stretch, which may result in a larger gauge factor compared with that for GF/Ag/PDMS-3. Then, above $25 \%$, a larger gauge factor was exhibited because of the dramatic increase in the cracks for the large strain, and a higher gauge factor was exhibited compared to that of other composites. In this way, GF/Ag/PDMS-3 exhibited negative curves (a smaller gauge factor) below $25 \%$ but positive curves above $25 \%$ (a larger gauge factor) compared to other strain sensors.
In addition to the high sensitivity, durability is another important index in the practical application of strain sensors, which can significantly reduce their cost and expand their application scope. The strain sensor was tested for durability at a speed of $1 \mathrm{~mm} \mathrm{~s}^{-1}$ for stretch cycles, and the results are shown in Fig. 6d-f. From the result of GF/Ag/PDMS-3 with a strain of $30 \%$, we can see that the relative resistance of the strain sensor varies greatly in the stretch-release process, and the stability of the sensor is excellent for 500 cycles. Fig. 6e represents several random stretching-relaxing cycles extracted from the red part in Fig. $6 \mathrm{~d}$, and the relative resistance change versus time curves exhibit great stability with high GF of 183 . The relative resistance change versus strain demonstrates negligible hysteresis, as shown in Fig. 6f, which endows the strain sensor with excellent stability and short response time.

The relative resistance change mechanism of the GF/AgNPs/ PDMS-based strain sensor with a "hollow packaged" structure is proposed and shown in Fig. 7. The high-aspect-ratio soft flexible rGO flakes can establish an effective electrical network between $\mathrm{Ag}$ NPs, and Ag NPs attached to the rGO flakes can promote the contact interface. The available sensitive signals can be enhanced by providing excellent electrical conductivity for electron capture and transport on the rGO flakes of the grown $\mathrm{Ag}$ NPs. In addition, the decoration of Ag nanoparticles significantly increases the conductivity of the 3D structure (Fig. 7a). When stretched with a small strain, the range of increase in resistance is slight due to the special "hollow packaged" structure. The synergistic conductive effects of the Ag NPs and the rGO flakes ( $<25 \%$ stretch strain) generate few cracks and at the same time, the 3D "core" of the GF/Ag composites is compressed along the vertical direction, which may increase some conductive paths, as shown in Fig. 7b. In this way, the comprehensive influence of the cracks coming from the stretch and the increase in the conductive paths during the compression was that the resistance changed slowly at the small strain $(<25 \%$ stretch strain). Then, with the continuous increase in the strain, the contact area between the layers of the rGO sheets and the Ag nanoparticles decreased dramatically and more cracks appeared along the horizontal direction. As a result, the resistance of the as-prepared strain sensors increased significantly with a dramatic response and high sensitivity (Fig. 7c).

The high sensitivity, large stretch range, and excellent durability make the as-prepared GF/Ag/PDMS strain sensor a high-performance wearable strain sensor to detect human motions, such as the bending and rotation of the wrist, finger bending, and arm bending. As shown in Fig. 8a, the relative resistance change increases significantly when the wrist bends and recovers to the original state. Fig. $8 \mathrm{~b}$ demonstrates the

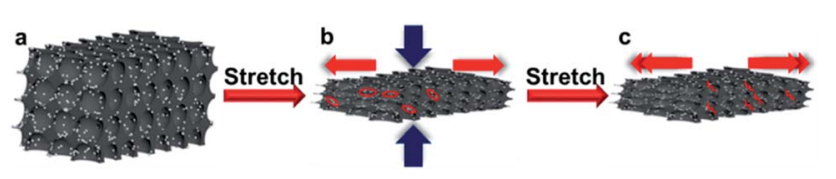

Fig. 7 Relative resistance change mechanism of GF/AgNPs/PDMSbased strain sensors with a "hollow packaged" structure: (a) original state, (b) under small strain, and (c) under large strain. 

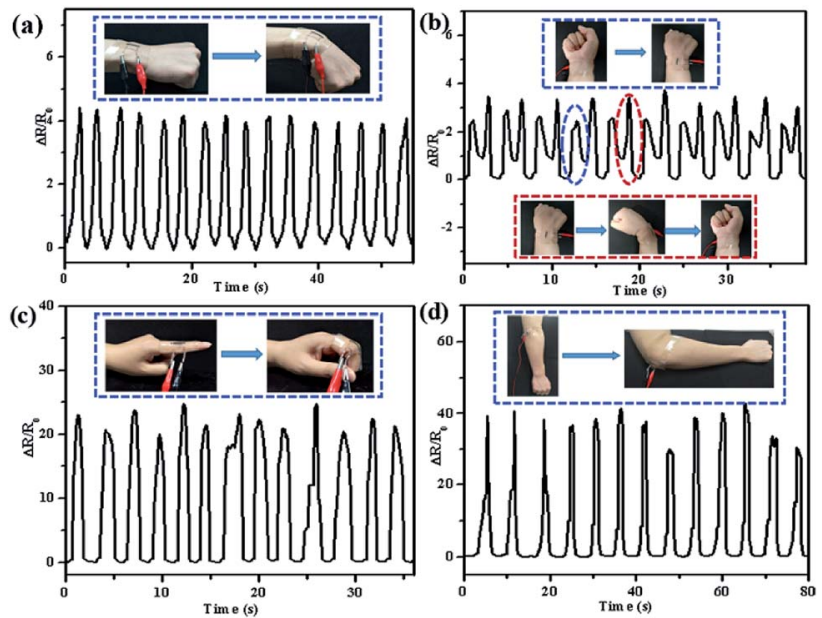

Fig. 8 Relative changes in resistance in the bending motion of (a) wrist bending, (b) wrist rotation (photographic images of the small rotation of the wrist are marked with a blue line, and the large rotation is marked with a red line), (c) finger bending, and (d) elbow bending.

relative change in resistance when the wrist is continuously rotated. The small rotation of the wrist is marked with a blue line, and the large rotation is marked with a red line. Therefore, the sensor can sense the bending and rotation of the wrist and can detect the movement of the wrist in practical applications. As shown in Fig. 8c, the as-prepared strain sensor can detect the change in relative resistance when the finger bends at $90^{\circ}$. It can be seen that the change in relative resistance is relatively stable and the sensitivity is relatively high. Fig. $8 \mathrm{~d}$ exhibits the detection of the change in relative resistance when the arm bends by $90^{\circ}$. The change in relative resistance is relatively stable. The strain produced by arm bending is slightly larger than that of finger bending, and the change in relative resistance is larger.

\section{Conclusion}

Highly sensitive and flexible strain sensors based on 3D graphene foams and Ag NPs with a special "hollow packaged" structure were fabricated by a simple method. Ag NPs were controllably reduced with PVP and ethanol at first and then assembled with GO sheets by ascorbic acid, forming the 3D GF/ $\mathrm{Ag}$ structures. Then, the freeze-dried GF-Ag samples were packaged with half-cured PDMS films, resulting in flexible strain sensors. The PDMS-encapsulated GF/Ag sensors exhibited high sensitivity with a gauge factor of 1588 (at a sensing strain of $50 \%$ ) and excellent stability in 500 cycles of $30 \%$ strain. Besides, the successful detection of the movements of humans such as bending of arms and fingers was demonstrated as well. Moreover, the mechanism of the "hollow packaged" sensor was proposed and the sensitivity of the strain sensor was improved by the synergistic action of Ag NPs and rGO. Considering the facile preparation process, high sensitivity and reproducibility as well as the successful detection of the motions of the human body, it is believed that the reported flexible sensor demonstrates a high potential for the application as a wearable strain sensor.

\section{Conflicts of interest}

There are no conflicts to declare.

\section{Acknowledgements}

This work was financially supported by R\&D Funds for Basic Research Program of Shenzhen (JCYJ20160331191741738), National Natural Science Foundation of China (61904191), China Postdoctoral Science Foundation (2018M640840), NSFCGuangdong Jointed Funding (U1601202), NSFC-Shenzhen Robot Jointed Funding (U1613215), Key Laboratory of Guangdong Province (2014B030301014), National Key R\&D Project from Minister of Science and Technology of China (2017ZX02519) and the Science and Technology Service Network Initiative of Chinese Academy of Sciences (KFJ-STS-SCYD-211).

\section{Notes and references}

1 M. Amjadi, K. U. Kyung, I. Park and M. Sitti, Adv. Funct. Mater., 2016, 26, 1678-1698.

2 K.-H. Kim, S. K. Hong, N.-S. Jang, S.-H. Ha, H. W. Lee and J.-M. Kim, ACS Appl. Mater. Interfaces, 2017, 9, 17499-17507.

3 S. J. Kim, W. Song, Y. Yi, B. K. Min, S. Mondal, K.-S. An and C.-G. Choi, ACS Appl. Mater. Interfaces, 2018, 10, 3921-3928.

4 S. R. Larimi, H. R. Nejad, M. Oyatsi, A. O'Brien, M. Hoorfar and H. Najjaran, Sens. Actuators, A, 2018, 271, 182-191.

5 T. Q. Trung and N. E. Lee, Adv. Mater., 2016, 28, 4338-4372. 6 M. Liao, P. Wan, J. Wen, M. Gong, X. Wu, Y. Wang, R. Shi and L. Zhang, Adv. Funct. Mater., 2017, 27, 1703852.

7 L. Yu, J. C. Yeo, R. H. Soon, T. Yeo, H. H. Lee and C. T. Lim, ACS Appl. Mater. Interfaces, 2018, 10, 12773-12780.

8 B. Liang, W. Chen, Z. He, R. Yang, Z. Lin, H. Du, Y. Shang, A. Cao, Z. Tang and X. Gui, Small, 2017, 13, 1702422.

9 Y. Li, J. Chen, L. Huang, C. Li, J. D. Hong and G. Shi, Adv. Mater., 2014, 26, 4789-4793.

10 H. Li, L. Jing, R. Y. Tay, S. H. Tsang, J. Lin, M. Zhu, F. N. Leong and E. H. T. Teo, Chem. Eng. J., 2017, 328, 825833.

11 X. Chang, S. Sun, S. Sun, T. Liu, X. Xiong, Y. Lei, L. Dong and Y. Yin, J. Alloys Compd., 2018, 738, 111-117.

12 Y. Zheng, Y. Li, K. Dai, Y. Wang, G. Zheng, C. Liu and C. Shen, Compos. Sci. Technol., 2018, 156, 276-286.

13 H. Xu, J. X. Xiang, Y. F. Lu, M. K. Zhang, J. J. Li, B. B. Gao, Y. J. Zhao and Z. Z. Gu, ACS Appl. Mater. Interfaces, 2018, 10, 11785-11793.

14 L. Jinlong, Y. Meng, K. Suzuki and H. Miura, Mater. Lett., 2017, 196, 369-372.

15 Y. J. Yun, J. Ju, J. H. Lee, S.-H. Moon, S.-J. Park, Y. H. Kim, W. G. Hong, D. H. Ha, H. Jang, G. H. Lee, H.-M. Chung, J. Choi, S. W. Nam, S.-H. Lee and Y. Jun, Adv. Funct. Mater., 2017, 27, 1701513.

16 K. S. Novoselov, V. I. Fal'ko, L. Colombo, P. R. Gellert, M. G. Schwab and K. Kim, Nature, 2012, 490, 192-200. 
17 A. K. Geim, science, 2009, 324, 1530-1534.

18 L. Embrey, P. Nautiyal, A. Loganathan, A. Idowu, B. Boesl and A. Agarwal, ACS Appl. Mater. Interfaces, 2017, 9, 3971739727.

19 Y. Gao, X. Fang, J. Tan, T. Lu, L. Pan and F. Xuan, Nanotechnology, 2018, 29, 235501.

20 Z. Chen, W. Ren, L. Gao, B. Liu, S. Pei and H.-M. Cheng, Nat. Mater., 2011, 10, 424.

21 Y. R. Jeong, H. Park, S. W. Jin, S. Y. Hong, S. S. Lee and J. S. Ha, Adv. Funct. Mater., 2015, 25, 4228-4236.

22 J. Li, S. Zhao, X. Zeng, W. Huang, Z. Gong, G. Zhang, R. Sun and C. P. Wong, ACS Appl. Mater. Interfaces, 2016, 8, 1895418961.

23 H. Song, S. Lei, X. Li, S. Guo, P. Cui, X. Wei, W. Liu and H. Liu, Sens. Actuators, A, 2018, 271, 53-59.

24 J. C. Y. Kenry and C. T. Lim, Microsyst. Nanoeng., 2016, 2, 16043.

25 S. Zhang, H. Zhang, G. Yao, F. Liao, M. Gao, Z. Huang, K. Li and Y. Lin, J. Alloys Compd., 2015, 652, 48-54.
26 H. Zhang, N. Liu, Y. Shi, W. Liu, Y. Yue, S. Wang, Y. Ma, L. Wen, L. Li, F. Long, Z. Zou and Y. Gao, ACS Appl. Mater. Interfaces, 2016, 8, 22374-22381.

27 Z. Yang, D. Y. Wang, Y. Pang, Y. X. Li, Q. Wang, T. Y. Zhang, J. B. Wang, X. Liu, Y. Y. Yang, J. M. Jian, M. Q. Jian, Y. Y. Zhang, Y. Yang and T. L. Ren, ACS Appl. Mater. Interfaces, 2018, 10, 3948-3954.

28 S. Zhao, L. Guo, J. Li, N. Li, G. Zhang, Y. Gao, J. Li, D. Cao, W. Wang, Y. Jin, R. Sun and C. P. Wong, Small, 2017, 13, 1700944.

29 J. Li, S. Zhao, G. Zhang, Y. Gao, L. Deng, R. Sun and C.-P. Wong, J. Mater. Chem. A, 2015, 3, 15482-15488.

30 D. Chen, X. Qiao, X. Qiu and J. Chen, J. Mater. Sci., 2009, 44, 1076-1081.

31 J. Zhang, H. Yang, G. Shen, P. Cheng, J. Zhang and S. Guo, Chem. Commun., 2010, 46, 1112-1114.

32 M. Chen, C. Zhang, X. Li, L. Zhang, Y. Ma, L. Zhang, X. Xu, F. Xia, W. Wang and J. Gao, J. Mater. Chem. A, 2013, 1, 2869. 